\title{
New Optimal Common-Mode Modulation for Three-Phase Inverters with DC-Link Referenced Output Filter
}

\author{
Michael Antivachis, Dominik Bortis, Ander Avila, and Johann W. Kolar
}

\begin{abstract}
A two-level inverter followed by a DC-link referenced output filter is a promising solution for high-speed low-voltage drive systems. A limitation of this power electronics topology, however lies in the high current ripple and hence additional losses occurring within the filter inductors. This shortcoming can be addressed by means of an appropriate modulation technique. This paper details two new modulation strategies, tailored to the specific characteristics of three-phase inverters with DC-link referenced output filter, utilizing the instantaneous common-mode (CM) voltage as a degree of freedom, in order to decrease the output inductor's current ripple. It is deduced that a constant (DC) CM injection modulation (DCCMM) or an AC CM injection modulation (ACCMM) with arbitrarily large amplitude yields the best performance depending on the operating point. The CM pattern that minimizes the inductor current ripple envelope results from the combination of the two schemes and constitutes the optimal CM injection modulation (OCMM). Furthermore, the proposed modulation schemes are tested on a $300 \mathrm{~W}$ hardware demonstrator driving a $300 \mathrm{krpm}$ custom designed motor, where a reduction of the total inverter losses of up to $11 \%$ is observed.
\end{abstract}

Index Terms-DC-link referenced output filter, high-speed drives, modulation scheme, optimal CM injection.

\section{INTRODUCTION}

$\mathrm{H}$ IGH-SPEED low-voltage (LV) drive systems spread across a wide range of applications such as turbocompressor systems, drills, medical equipment and air-conditioning units [1], [2]. Typically, a two-level pulse width modulated (PWM) inverter with high switching frequency $f_{\mathrm{s}}$ is preferred in order to provide the motor with a high quality sinusoidal current that minimizes the induced machine losses (cf. Fig. 1(a)). The insertion of an AC differential-mode (DM) interface filter between the converter and the machine further smoothes the motor terminal currents and voltages [3]. An AC-side common-mode (CM) filter suppresses the high-frequency $\mathrm{CM}$ voltage applied to the machine terminals and hence protects the motor insulation and bearings from overvoltage stress [4]. Moreover, the CM filter is an effective measure against conducted EMI noise, since it provides to

Manuscript received November 19, 2017

M. Antivachis, D. Bortis and J. W. Kolar are with the Power Electronic Systems Laboratory, ETH Zurich, Switzerland (e-mail: antivachis@lem.ee.ethz. ch). A. Avila is with the Power Electronics Area, IK4-Ikerlan Technology Research Centre, Spain (e-mail: aavila@ikerlan.es).

Digital Object Identifier 10.24295/CPSSTPEA.2017.00030

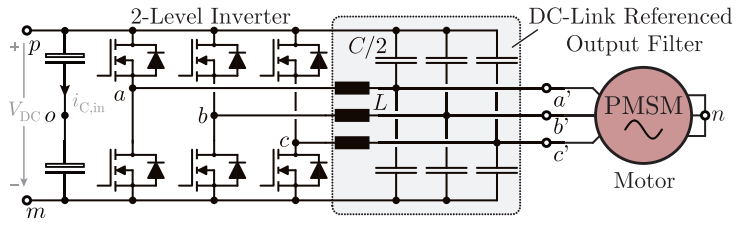

(a)

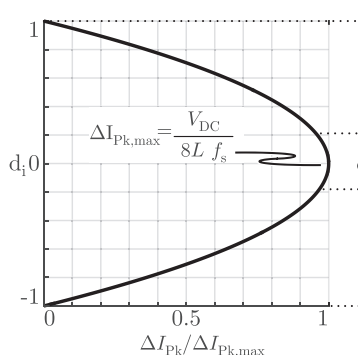

(b)

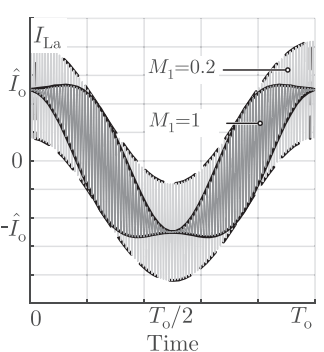

(d)

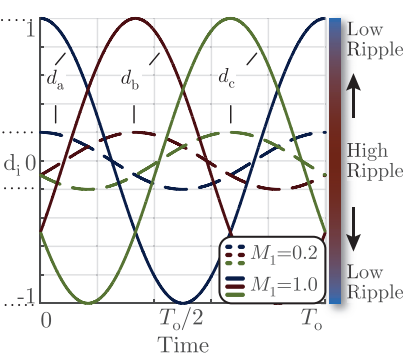

(c)

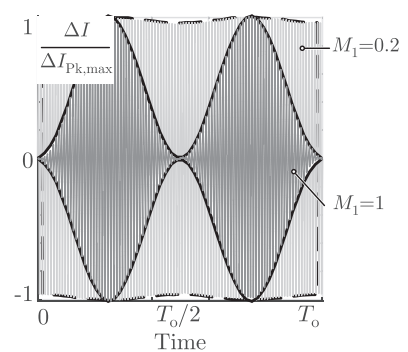

(e)
Fig. 1. In (a) the schematic of a two-level inverter with a DC-link referenced output filter followed by a high-speed motor is depicted. In (b) the correlation between the normalized peak current ripple $\Delta I_{\mathrm{Pk}}$ and the instantaneous duty cycle $d_{\mathrm{i}}$ is presented. In (c) the sinusoidal duty cycles for two different modulation depths are shown, while in (d) and (e) the resulting inductor currents and current ripples of phase $a$ for the different modulation depths $M_{1}$ are illustrated respectively.

the CM currents, induced by the switching operation of the converter, a low impedance path that confines the CM noise within the inverter stage. The DC-link referenced output filter, presented in Fig. 1(a), performs simultaneously CM and DM attenuation [5]-[7] and thus is a prominent choice for LV drive systems.

Accordingly, the incorporation of a DC-link referenced output filter allows for higher motor efficiency and CM noise suppression, at the expense of decreased converter performance, since the volume and losses of the output filter are added to 
the converter stage. There has been extensive research towards counterbalancing the negative impact of the output filter by means of appropriate inverter modulation techniques [8]-[11]. For three-phase inverters employing carrier-based PWM techniques, the available degree of freedom is the shape of the injected CM duty cycle $d_{\mathrm{CM}}(t)$ [12]. Hence, the duty cycles of the three phases can be written as

$$
\begin{aligned}
& d_{\mathrm{a}}(t)=M_{1} \cos (\omega t)+d_{\mathrm{CM}}(t) \\
& d_{\mathrm{b}}(t)=M_{1} \cos \left(\omega t-\frac{2 \pi}{3}\right)+d_{\mathrm{CM}}(t) \\
& d_{\mathrm{c}}(t)=M_{1} \cos \left(\omega t+\frac{2 \pi}{3}\right)+d_{\mathrm{CM}}(t)
\end{aligned}
$$

where $M_{1}$ is the modulation depth defined as $M_{1}=\frac{2 \hat{V}_{\mathrm{o}}}{V_{\mathrm{DC}}}$ and $\hat{V}_{\mathrm{o}}$ is the peak output phase voltage. Furthermore, the local average of the inverter switched voltages is given as $\left\langle v_{i, o}\right\rangle_{T_{s}}=$ $d_{\mathrm{i}}(t) \frac{V_{\mathrm{DC}}}{2}$, where $i \in\{a, b, c\}$.

The aim of the CM injection with duty cycle $d_{\mathrm{CM}}(t)$ is the minimization of the current ripple across the inductor and hence the mitigation of the inductor related losses. For example, in the case of a two-level inverter followed by an exclusive DM filter, the injection of an approximately triangular CM pattern yields the lowest filter inductor current ripple (standard space vector modulation - SVM) [13], while a sinusoidal third harmonic injection (THM) results in similarly high inductor performance. Moreover, the injection of a roughly rectangular CM component, known in the literature also as discontinuous modulation (DCM) [14], [15], leads to a reduction of the switching losses. However, in the case of a two-level inverter with a DC-link referenced output filter, the formulation mechanism of the filter inductor current $i_{\mathrm{L}}$ is fundamentally different compared to a simple DM filter, because high-frequency currents are allowed to flow through the DC-link connected capacitors $C / 2$ from/towards the DClink. Thus, the traditional modulation techniques do not yield anymore the desired performance gain. Instead, the optimal shape of injected CM duty cycle $d_{\mathrm{CM}}(t)$, that minimizes the current ripple in the filter inductor, must be revisited and redefined according to the special characteristics of the filter topology at hand.

In response to those concerns, this paper details the degrees of freedom when selecting a modulation scheme and proposes two modulation techniques that reduce the filter inductor current ripple. In a first step, the constant CM shifting (DCCMM) of all three phases $d_{\mathrm{CM}}(t)=M_{0}$ is presented, and subsequently the injection of a AC CM pattern (ACCMM) with arbitrarily large amplitude $d_{\mathrm{CM}}(t)=-M_{\mathrm{N}} \cos (N \omega t)(\mathrm{N}=$ $3,6,9, \ldots)$ is examined. The inductor current ripple formulation and trade-offs between the two modulation schemes are analyzed and quantified in Sec. II. Afterwards, the achievable system performance, in terms of inductor RMS current ripple, is evaluated by means of a multi-objective optimization. Based on the results, the optimal CM injection modulation scheme (OCMM) is proposed. In Sec. III, the claimed performance benefit is validated against experimental mea- surements and finally the conclusions are drawn in Sec. IV.

\section{Generalized CM Injection Modulation}

First, the current ripple formation of the filter inductor in the case of a two-level inverter with a DC-link referenced output filter is analyzed. The standard modulation case, where all duty cycles are purely sinusoidal (sinusoidal modulation - SM), i.e. without any CM pattern injection $\left(d_{\mathrm{CM}}(t)=\right.$ $0)$, is considered (Fig. 1(c)). The switched-node voltage $v_{\text {ao }}(t)$ of phase $a$ with respect to the DC-link midpoint $o$, contains a fundamental component, as well as high-frequency harmonics concentrated around and above the switching frequency $f_{\mathrm{s}}$ (cf. Fig. 2(a)). This switched voltage, with voltage levels between $+V_{\mathrm{DC}} / 2$ and $-V_{\mathrm{DC}} / 2$, is subsequently smoothed by the output filter and results in a predominantly sinusoidal terminal voltage $v_{\mathrm{a}^{\prime} \mathrm{n}}=M_{1} \cos (\omega t)$, with respect to the load open star-point $n$. Moreover, due to the connection of the output filter capacitors $(C / 2)$ to the positive and negative DC rails $(p, m)$, a stable sinusoidal terminal voltage $v_{\mathrm{a}, \mathrm{o}}=M_{1} \cos (\omega t)$ with respect to the DC-link midpoint $o$ is also generated. The difference between the switch node voltage $v_{\text {ao }}$ and the terminal voltage $v_{\mathrm{a}}$ o yields the current ripple inducing voltage across the inductor $v_{\mathrm{La}}=v_{\mathrm{ao}}-v_{\mathrm{a}, \mathrm{o}}$. The inductor voltage $v_{\mathrm{La}}$ is clearly a two-level voltage and generates an inductor current ripple similar to the case of a single-phase inverter. Due to the DC-link referenced output filter, the current ripple of each phase $\Delta I_{\mathrm{Pk}}$ is independent of the remaining two phases (cf. Fig. 1(b)) and is determined by the duty cycle $d_{\mathrm{i}}(\mathrm{t})$ of the corresponding phase $i \in a, b, c$ as

$$
\begin{aligned}
& \Delta I_{\mathrm{Pk}}=\frac{\left(1+d_{\mathrm{i}}\right)\left(1-d_{\mathrm{i}}\right) V_{\mathrm{DC}}}{8 f_{\mathrm{s}} L} \\
& \Delta I_{\mathrm{RMS}}=\frac{\Delta I_{\mathrm{Pk}}}{\sqrt{3}}=\frac{\left(1+d_{\mathrm{i}}\right)\left(1-d_{\mathrm{i}}\right) V_{\mathrm{DC}}}{8 \sqrt{3} f_{\mathrm{s}} L}
\end{aligned}
$$

where $d_{\mathrm{i}} \in[-1,1]$.

If a triangular carrier signal is defined in the interval $[-1$, 1], the duty cycle that maximizes the instantaneous inductor current ripple $\Delta I_{\mathrm{Pk}}$ is $d_{\mathrm{i}}=0$. The corresponding peak and RMS current ripple over a switching period are then calculated as

$$
\begin{aligned}
& \Delta I_{\mathrm{Pk}, \text { max }}=\frac{V_{\mathrm{DC}}}{8 f_{\mathrm{s}} L} \\
& \Delta I_{\mathrm{RMS}, \max }=\frac{\Delta I_{\mathrm{Pk}, \text { max }}}{\sqrt{3}}=\frac{V_{\mathrm{DC}}}{8 \sqrt{3} f_{\mathrm{s}} L}
\end{aligned}
$$

and illustrated in Fig. 1(b).

In contrast, the current ripple is zero when the duty cycle is in the vicinity of 1 or -1 . For low modulation depths $M_{1}$, the duty cycles of all three phases remain close to the zero line for the whole fundamental period $T_{\mathrm{o}}$. As a results, the current ripple and inductor losses are maximized when the modulation depth, and hence the machine induced voltage, speed and power are low (Fig. 1(c)-(e), $M_{1}=0.2$ ). Conse- 


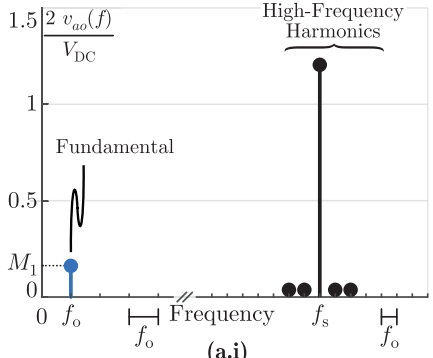

(a.i)

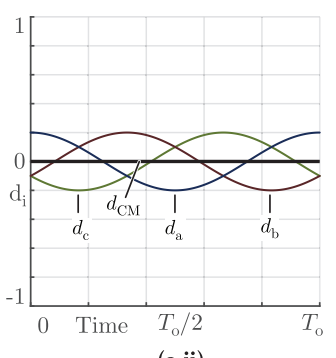

(a.ii)

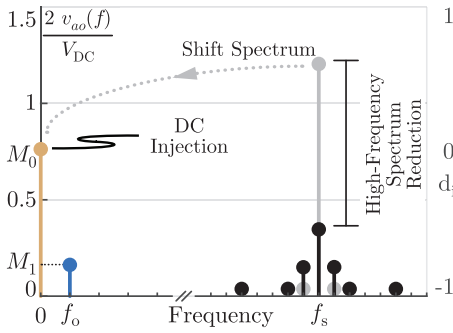

(b.i)

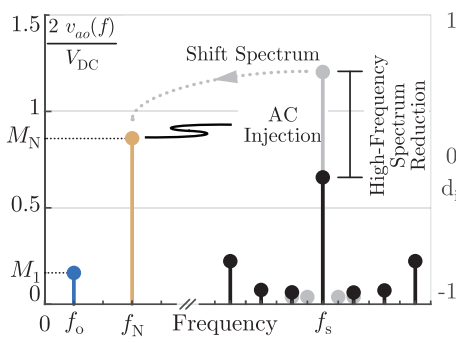

(c.i)

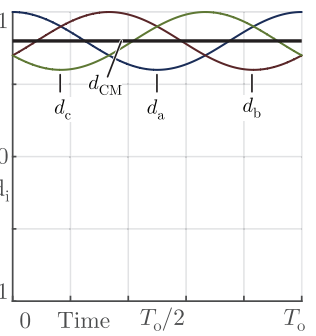

(b.ii)

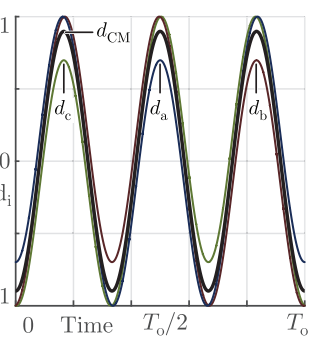

(c.ii)
Fig. 2. In (a.i), the spectrum of the inverter switch node voltage $v_{\mathrm{ao}}$ is shown when the standard sinusoidal modulation (SM) of (a.ii) is employed. (b.ii) illustrates the DCCMM technique, where all the duty cycles are shifted upwards by a DC voltage component $v_{\mathrm{CM}}=d_{\mathrm{CM}} \frac{V_{\mathrm{DC}}}{2}$, where $d_{\mathrm{CM}}=M_{0}$. The resulting advantageous spectral shaping of the inverter voltage is depicted in (b.i), where spectral content is transferred from the switching frequency $f_{\mathrm{s}}$ to the DC component. In (c.i), the qualitative inverter voltage spectrum distribution is illustrated, when ACCMM (c.ii) modulation is employed. In this case, an AC CM component is injected to the three phase duty cycles leading to a reduction of the high frequency voltage spectrum, whereas a part of the spectral content around the switching frequency $f_{\mathrm{s}}$ is shifted to the frequency of the CM component $f_{\mathrm{N}}$.

quently, for the standard modulation (i.e. no CM injection), the low load and part load efficiency deteriorates due to the high ratio between the losses induced within the filter inductor and the low output power. It becomes evident that steering away from the unfavorable duty cycle $d_{\mathrm{i}}=0$ is a path towards superior inductor performance. This can be achieved by means of CM injection, meaning that an identical signal $d_{\mathrm{CM}}(t)$, either constant or variable, is added to the duty cycles of all three phases as shown in (1). This CM signal $v_{\mathrm{CM}}=v_{\mathrm{no}}=d_{\mathrm{CM}} \frac{V_{\mathrm{DC}}}{2}$ shifts the voltage of all three inverter terminal nodes either higher or lower with respect to the DC-link midpoint $o$. If examined from the frequency domain standpoint, the injection of a low-frequency CM signal advantageously shapes the spectral content of the inverter

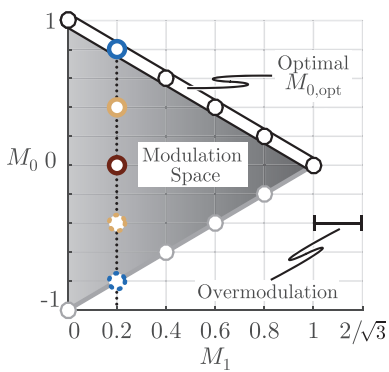

(a)

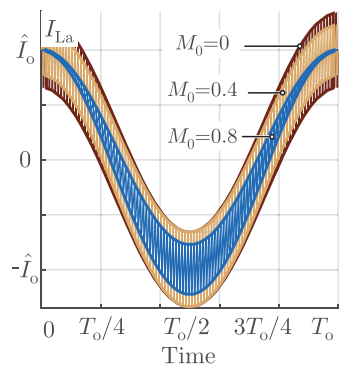

(c)

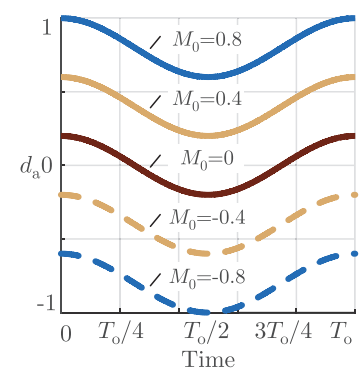

(b)

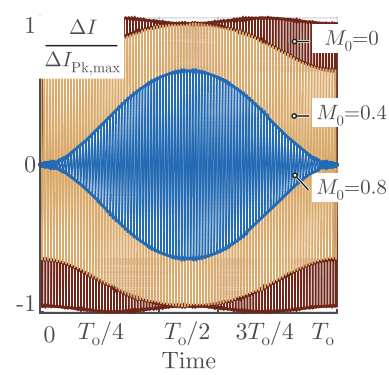

(d)
Fig. 3. Constant CM shifting (DCCMM) overview. In (a), the available $M_{0}$ values depending on the modulation depth $M_{1}$ are depicted. In (b), the effect of different values of $M_{0}$ on the duty cycle of phase $a$ for a constant modulation index $M_{1}=0.2$ is visualized. In (c) and (d), the impact of the DCCMM modulation technique on the inductor current and the current ripple is shown respectively.

switch node voltage $v_{\mathrm{i}, 0}, i \in a, b, c$, i.e. energy is transferred from the switching frequency harmonics to the low-frequency component corresponding to the injected CM pattern (cf. Fig. 2(b.i)-(c.i)). Hence, the current ripple inducing highfrequency voltage harmonics is limited and accordingly the output filter inductor efficiency is increased. Two CM signal types are proposed and explained within the remainder of the chapter: a positive or negative constant shifting (DCCMM) (cf. Fig. 2(b.i)-(b.ii)) and an AC CM pattern (ACCMM) (cf. Fig. 2(c.i)-(c.ii)) with arbitrarily large amplitude. Both options are compared against the standard modulation scheme (SM) with purely sinusoidal duty cycles, in terms of inductor current ripple.

\section{A. DC CM Modulation (DCCMM)}

The constant CM injection strategy (DCCMM) utilizes a constant CM signal $d_{\mathrm{CM}}=M_{0}$ that shifts all instantaneous sinusoidal duty cycle signals $d_{\mathrm{i}}$ away from the high current ripple region around $d_{\mathrm{i}}=0$ towards a lower ripple zone close to either $d_{\mathrm{i}}=1$ or $d_{\mathrm{i}}=-1$. Hence, the $\mathrm{CM}$ shifting can be either positive or negative, with symmetric effects on the inductor current ripple. The constant $\mathrm{CM}$ shifting technique is visualized in Fig. 3(b) for the example with modulation depth $M_{1}=0.2$. For a given modulation depth $M_{1}$, the acceptable range of the $M_{0}$ injection must be defined. This can be easily derived by the constraint that all the duty cycle signals must not exceed the carrier boundaries (i.e. $-1 \leq d_{\mathrm{i}} \leq 1$ ). Depending on the modulation depth $M_{1}$, the $M_{0}$ injection limits 
are then calculated by

$$
\begin{aligned}
& M_{1} \in[0,1], d_{\mathrm{CM}}(t)=M_{0} \\
& M_{0} \in\left[M_{0, \min }, M_{0, \max }\right], \\
& \text { where: } \quad M_{0, \min }\left(M_{1}\right)=-\left(1-M_{1}\right) \\
& M_{0, \max }\left(M_{1}\right)=\left(1-M_{1}\right),
\end{aligned}
$$

and the available modulation space for $M_{0}$ is visualized in Fig. 3(a). It should be noted that the feasible $M_{0}$ interval becomes wider for low modulation depths $M_{1}$ : for instance when $M_{1}=0.2$ (cf. Fig. 3), the $M_{0}$ parameter can be freely selected within the interval $[-0.8,+0.8]$. In contrast, for $M_{1}=1$, the $M_{0}$ value must be compulsively kept at zero, otherwise the instantaneous duty cycles would exceed the carrier boundaries. When $M_{1}>1$ (i.e. overmodulation), the DCCMM technique is impractical, since it provokes undesired pulse dropping which in return dramatically increases the inductor current ripple. In other words, the DCCMM scheme is only advisable for the linear modulation region, i.e. $M_{1} \in[0,1]$.

The effect of the CM injection on the current envelope is profound, which means that the current ripple envelope is contracting with increasing CM injection $M_{0}$ (cf. Fig. 3(c)), thus the CM shifting positively impacts on the inductor performance. For the simple sinusoidal modulation (SM), the current ripple reaches its maximum at the zero crossings of the duty cycle which is at the time instants $t=\frac{T_{0}}{4}$ and $\frac{3 T_{0}}{4}$ (cf. Fig. 3(d), $M_{0}=0$ ). However, if a constant CM shifting is applied, the time instances of the zero crossing are changing. This means that for $0<M_{0}<M_{1}$ the two zero crossings are approaching $\mathrm{t}=\frac{T_{0}}{2}$, while for $0<M_{1}<M_{0}$ no zero crossings take place and the maximum current ripple is solely found at $t=\frac{T_{0}}{2}$ (cf. Fig. 3(d) for $M_{0}=0.4$ or $M_{0}=0.8$ ).

\section{B. AC CM Modulation (ACCMM)}

In a second step, an alternative CM injection technique is examined which employs a sinusoidal AC CM pattern $d_{\mathrm{CM}}(t)$ $=-M_{\mathrm{N}} \cos \left(2 \pi f_{\mathrm{N}} t\right)$ with a frequency $f_{\mathrm{CM}}=f_{\mathrm{N}}=N f_{\mathrm{o}}$ and an amplitude $M_{\mathrm{N}}$. By choosing a large amplitude $M_{\mathrm{N}}$, the highfrequency spectrum of the switch node voltage $v_{\mathrm{i}, \mathrm{o}}$ is reduced and instead more spectral content is concentrated at the CM signal frequency $f_{\mathrm{N}}$ (cf. Fig. 2(c)). Hence, the high-frequency current ripple is reduced and superior performance in terms of losses can be achieved. Special attention should be paid to the reactive phase current flowing through the filter capacitors, since the $N^{\text {th }}$ order harmonic current $\hat{I}_{\mathrm{C}, \mathrm{N}}$, calculated as

$$
\hat{I}_{\mathrm{C}, \mathrm{N}}=M_{\mathrm{N}} V_{\mathrm{DC}} \pi N f_{\mathrm{o}} C
$$

is proportional to the amplitude $M_{\mathrm{N}}$ and the frequency $f_{\mathrm{N}}$ of the AC harmonic voltage. Especially for high speed motor drives, where the fundamental frequency $f_{\mathrm{o}}$ can be in the $\mathrm{kHz}$-range, the reactive currents at the frequency $f_{\mathrm{N}}=\mathrm{N} \cdot f_{\mathrm{o}}$ become significant and hence should be limited. Otherwise additional conduction losses are occurring in the inductors and semiconductor devices, degrading the overall system performance. In the course of this research, which focuses on ultra-high speed drive systems, the frequency of the AC CM signal must be set to the lowest possible $\mathrm{CM}$ frequency $f_{\mathrm{CM}}$ $=3 f_{0}$, thus the ACCMM reduces to a generalized third harmonic modulation scheme (GTHM). According to the conventional third harmonic injection (THM), the amplitude of the third harmonic, $M_{3}$, is typically set to $\frac{M_{1}}{6}$ or $\frac{M_{1}}{4}$ in order to allow linear overmodulation (i.e. $M_{1} \in\left[1, \frac{2}{\sqrt{3}}\right]$ ). In contrast, for the proposed generalized third harmonic modulation scheme (GTHM), the amplitude of the third harmonic $M_{3}$ is considered as a degree of freedom that can be set arbitrarily. The GTHM clearly constitutes an extension of the conventional third harmonic modulation, thus both are applicable in the overmodulation region (i.e. $M_{1} \in\left[1, \frac{2}{\sqrt{3}}\right]$ ).

Depending on the modulation depth $M_{1}$, the maximum and minimum allowable value of third harmonic injection $M_{3}$ must be defined in a similar fashion as in the case of DCCMM, i.e. the modulation index is constrained within $-1 \leq$ $d_{\mathrm{i}} \leq 1$. The closed form solution can be found as

$$
\begin{aligned}
& M_{1} \in\left[0, \frac{2}{\sqrt{3}}\right], d_{\mathrm{CM}}(t)=-M_{3} \cos (3 \omega t) \\
& M_{3} \in\left[M_{3, \min }, M_{3, \max }\right] \\
& \text { where: } M_{3, \min }\left(M_{1}\right)=-\left(1-M_{1}\right) \\
& \qquad M_{3, \max }\left(M_{1}\right):\left\{\begin{array}{l}
\text { Solving nonlinear equation } \\
M_{1} \cos (\phi)-M_{3, \max } \cos (3 \phi)=1 \\
\phi=\sin ^{-1}\left(\sqrt{\frac{9 M_{3, \max }-M_{1}}{12 M_{3, \max }}}\right)
\end{array}\right.
\end{aligned}
$$

The corresponding modulation area, which is asymmetric, is depicted in Fig. 4(a). In Fig. 4(b), third harmonic patterns with different amplitudes are superimposed on the sinusoidal duty cycle of phase $a$, for the example of modulation depth $M_{1}=0.2$. As can be noticed, the generalized third harmonic injection shapes the current ripple envelope in a way that it is continuously alternating between high and low current ripple (cf. Fig. 4(c)) whereas the inductor current stress decreases, with increasing $M_{3}$ values (cf. Fig. 4(d)). In analogy to DCCMM, it should be noted that for GTHM the available range of $M_{3}$ values is wide for low modulation depths $M_{1}$, and for high modulation depths the $M_{3}$ parameter is increasingly restricted.

\section{Optimal CM Modulation (OCMM)}

The idea behind the two modulation schemes, constant CM shifting (DCCMM) and generalized third harmonic injection (GTHM) is now established. The corresponding parameters $M_{0}$ and $M_{3}$ constitute degrees of freedom and must be selected in a way that minimizes the inductor current stress. From a numerical optimization standpoint, the mod- 


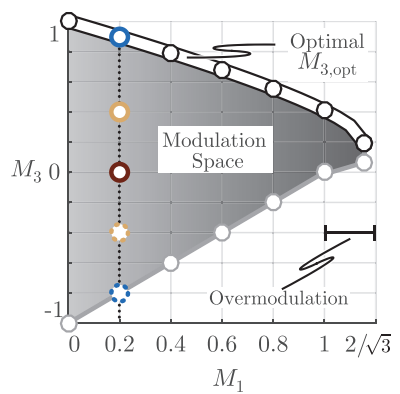

(a)

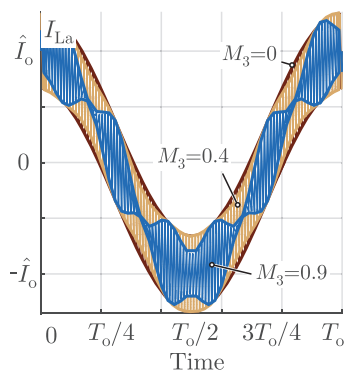

(c)

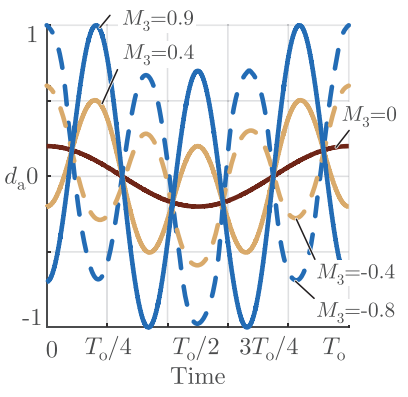

(b)

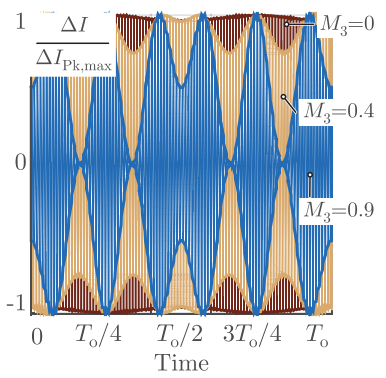

(d)
Fig. 4. Generalized third harmonic injection (GTHM) overview. In (a), the allowable $M_{3}$ values depending on the modulation depth $M_{1}$ are depicted. In (b), the effect of different values of $M_{3}$ on the duty cycle of phase $a$ for a constant modulation index $M_{1}=0.2$ is visualized. In (c) and (d), the impact of the GTHM modulation technique on the inductor current and the current ripple is shown respectively.

ulation parameters $M_{0}$ and $M_{3}$, which are subject to linear constraints $M_{0, \text { min }}<M_{0}<M_{0, \text { max }}$ and $M_{3, \text { min }}<M_{3}<M_{3, \text { max }}$, represent the optimization variables and have to be optimized in such a way that for a given modulation depth $M_{1}$ the optimal CM values $M_{0, \text { opt }}$ and $M_{3, \text { opt }}$, resulting in the lowest inductor RMS current ripple, are found. Accordingly, the objective function $F$ is defined as the normalized inductor RMS current ripple over a fundamental period $T_{\mathrm{o}}$ and quantifies the effectiveness of the employed parameter combination of $M_{0}$ and $M_{3}$,

$$
F=\frac{\Delta I_{\mathrm{RMS}}}{\Delta I_{\mathrm{RMS}, \max }}=\frac{\sqrt{\frac{1}{T_{\mathrm{o}}} \int_{0}^{T_{\mathrm{o}}} \Delta I_{\mathrm{L}, \mathrm{a}}^{2}(t) d t}}{\frac{V_{\mathrm{DC}}}{8 \sqrt{3} f_{\mathrm{s}} L}} .
$$

The goal of the optimization algorithm is to identify the optimal set of parameters $M_{0}$, and $M_{3}$, such that the objective function $F$ is minimized.

The optimization procedure for the parameter $M_{0}$ of the constant $\mathrm{CM}$ injection techniques is analyzed first. For one modulation depth $M_{1}$ (cf. Fig. 3(b) with e.g. $M_{1}=0.2$ ), the acceptable $M_{0}$ parameter values (in this case $M_{0} \in[-0.8,+0.8]$ ) are swept and for each $M_{0}$ case the resulting normalized RMS current ripple $F\left(M_{1}, M_{0}\right)$ is calculated. Subsequently, the $M_{0}$ value that results in the minimization of the objective function (i.e. minimum current ripple) is selected as optimal modulation choice $M_{0, \text { opt }}\left(M_{1}\right)$ (cf. Fig. 3(c),(d), $M_{0, \text { opt }}(0.2)=$ $0.8)$. The process is iterated for all the modulation depths $M_{1}$ $\in[0,1]$ as visualized in Fig. 5(a), and thereby the optimal value of the constant CM shifting scheme (DCCMM) is derived. It should be noted that the constant $\mathrm{CM}$ injection scheme is symmetric in the sense that identical performance is attained by shifting upwards by $+M_{0}$ or downwards by $-M_{0}$, while the optimization reveals that the largest $\left|M_{0}\right|$ leads to the lowest inductor current ripple. The optimal values of $M_{0, \text { opt }}$ depending on the modulation depth $M_{1}$ are highlighted in Fig. 3(a), while Fig. 5(c) shows how the current ripple for the optimized DCCMM technique in comparison to the standard sinusoidal modulation (SM) evolves over different operating points.

The same optimization procedure used for $M_{0}$ can be applied for the optimization of the parameter $M_{3}$ of the GTHM modulation scheme. The optimal amplitude $M_{3, \mathrm{opt}}$ of the third harmonic that yields the lowest current ripple (i.e. minimizes $\left.F\left(M_{1}, M_{3}\right)\right)$ is calculated for each modulation index $M_{1}$ and thus the optimal third harmonic injection modulation scheme is derived (cf. Fig. 5(b)). In this case, the inductor current ripple changes in an asymmetric fashion with respect to $M_{3}$. Indeed, the amplitudes of the third harmonic $+M_{3}$ and $-M_{3}$ (negative amplitude indicates phase shift of $180^{\circ}$ ) result in different inductor performance. In analogy to the DCCMM, the optimization deduces that the maximum positive amplitude of third harmonic always yields the best inductor performance as visualized in Fig. 4(a). A key feature of the GTHM is that in contrast to the DCCMM it can also be used in the over modulation region $M_{1} \in\left[1, \frac{2}{\sqrt{3}}\right]$. The minimum current ripple achievable by employing GTHM over the whole converter operation range is plotted in Fig. 5(c), and is compared against the corresponding performance of the sinusoidal modulation (SM).

A possible combination of two $\mathrm{CM}$ injection patterns (DCCMM, GTHM) is also investigated. In this case, the inserted CM pattern is $d_{\mathrm{CM}}=M_{0}-M_{3} \cos (3 \omega t)$. The results reveal that merging both modulations (i.e. simultaneously $M_{0}$ $\neq 0$ and $M_{3} \neq 0$ ) does not yield superior performance in terms of current ripple. Instead for low modulation indexes $M_{1}<0.5$ the exclusive utilization of constant CM shifting (DCCMM) (i.e. $M_{0} \neq 0, M_{3}=0$ ) allows for the smallest current ripple, while for high duty cycles $M_{1}>0.5$ the generalized third harmonic injections (GTHM) is more effective (i.e. $M_{0}=0, M_{3}$ $\neq 0$ ). The modulation scheme that transitions from DCCMM (below $M_{1}=0.5$ ) to GTHM (above $M_{1}=0.5$ ) constitutes the optimal CM injection modulation (OCMM) as indicated in (Fig. 5(c)) and minimizes the inductor losses over the whole inverter operating range. At the transition point $M_{1}=0.5$, the parameter $M_{0}$ must be decreased while the parameter $M_{3}$ must be increased in a way such that all the duty cycles remain within the carrier limits, and that the output DC-link referenced filter is not excited. To this end a smooth transition is proposed according to which the values of $M_{0}$ and $M_{3}$ are progressively adjusted within a transition interval $M_{1} \in$ $[0.4,0.6]$ around the transition point $M_{1}=0.5$. Namely, the parameter $M_{0}$ is linearly decreased with respect to $M_{1}$, from $M_{0}=0.6$ at $M_{1}=0.4$ to $M_{0}=0$ at $M_{1}=0.6$. Accordingly the parameter $M_{3}$ is increased from $M_{3}=0$ at $M_{1}=0.4$ to $M_{3}=$ 


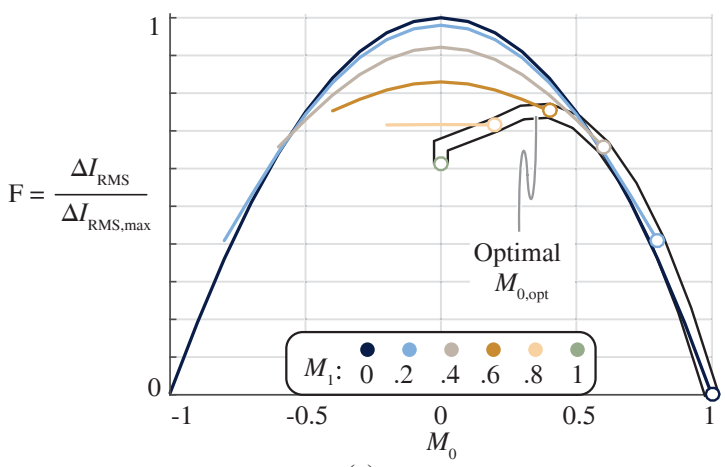

(a)

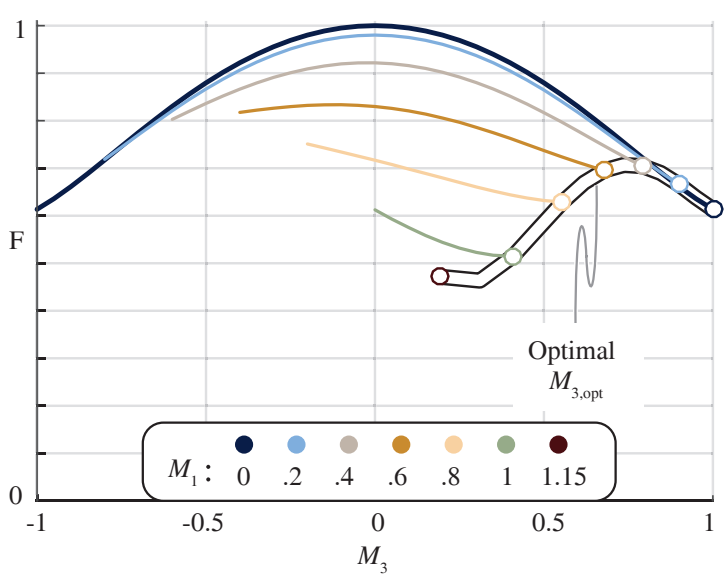

(b)

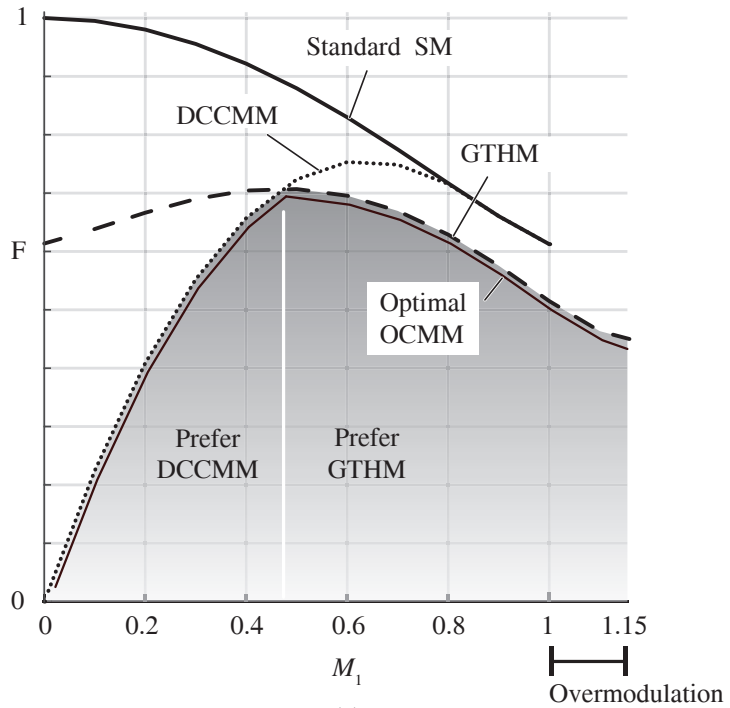

(c)

Fig. 5. Optimization procedure of the DCCMM and GTHM modulation parameters $M_{0}$ and $M_{3}$. In (a), the DCCMM strategy is analyzed: for each modulation depth $M_{1}$ all the possible values of the optimization parameter $M_{0}$ are sweped and the corresponding normalized current ripple is calculated for each value. The $M_{0, \text { opt }}$ value that yields the lowest RMS inductor current ripple $F$ (cf. (7)) constitutes the optimal choice and is highlighted. In (b), the same optimization method is visualized for the case of GTHM and the parameter value $M_{3}$. The optimal set of parameters $M_{0, \mathrm{opt}}$ and $M_{3, \mathrm{opt}}$ for each modulation depth $M_{1}$ are visualized in Fig. 3(a) and Fig. 4(a) respectively. Finally, in (c), the performance of the optimized DCCMM and GTHM, in terms of current ripple, is depicted and is compared against the standard sinusoidal modulation (SM).
0.68 at $M_{1}=0.6$. The proposed transition strategy ensures uninterrupted inverter operation at the expense of increased current ripple (suboptimal selection of $M_{0}, M_{3}$ in the transition region).

\section{Inverter Component Stress}

In order to provide a comprehensive overview of the total inverter performance when the OCMM is employed, the stresses on the remaining converter components (i.e. DClink capacitor, power semiconductors) are derived by means of analytic closed form expressions. There, a sinusoidal output inverter current $I_{\mathrm{L}, \mathrm{a}}=\hat{I}_{\mathrm{o}} \cos \left(\omega_{\mathrm{o}} t-\Phi\right)$ (i.e. current ripple is neglected), slightly lagging the motor AC voltage $v_{\mathrm{a}^{\prime} \mathrm{n}}=M_{1}$ $\frac{V_{\mathrm{DC}}}{2} \cos \left(\omega_{\mathrm{o}} t\right)$ by the phase angle $\Phi$ (i.e. low reactive power consumption), is assumed in the course of the calculations [16].

For the stress analysis of the power semiconductors, first the RMS current stress of the top- and low-side devices of phaseleg $a$ are examined. The same consideration can be extended to the remaining two phases due to symmetry. The local RMS current (i.e. over one switching period $T_{\mathrm{s}}$ ) of the low and high side switches of phase-leg $a$ are calculated as follows,

$$
\begin{aligned}
& \left\langle I_{\mathrm{a}, \text { High,RMS }}(t)\right\rangle=\sqrt{\frac{1+d_{\mathrm{a}}(t)}{2}} \cdot I_{\mathrm{La}}(t), \\
& \left\langle I_{\mathrm{a}, \text { Low }, \text { RMS }}(t)\right\rangle=\sqrt{\frac{1-d_{\mathrm{a}}(t)}{2}} \cdot I_{\mathrm{La}}(t),
\end{aligned}
$$

where $d_{\mathrm{a}}(t)=M_{1} \cos (\omega t)+M_{0}-M_{3} \cos (3 \omega t)$.

Based on the local RMS values, the global RMS current stress over a fundamental period $T_{\mathrm{o}}$ can be calculated as

$$
\begin{aligned}
& I_{\mathrm{a}, \text { High,RMS }}=\sqrt{\frac{1}{T_{\mathrm{o}}} \int_{0}^{T_{\mathrm{o}}}\left\langle I_{\mathrm{a}, \text { High,RMS }}(t)\right\rangle^{2} d t}=\hat{I}_{\mathrm{o}} \sqrt{\frac{1+M_{0}}{4}}, \\
& I_{\mathrm{a}, \text { Low }, \text { RMS }}=\sqrt{\frac{1}{T_{\mathrm{o}}} \int_{0}^{T_{\mathrm{o}}}\left\langle I_{\mathrm{a}, \text { Low }, \text { RMS }}(t)\right\rangle^{2} d t}=\hat{I}_{\mathrm{o}} \sqrt{\frac{1-M_{0}}{4}} .
\end{aligned}
$$

As can be noted from (9), in the case of DCCMM (i.e. $M_{0}$ $\neq 0, M_{3}=0$ ), the RMS current burden is unequal between the top- and low-side switch. For example, when positive DC CM shifting $\left(M_{0}>0\right)$ is selected, the duty cycles of all three phases are shifted upwards and therefore the relative on-time of the high-side semiconductors is longer than the corresponding on-time of the low-side devices. Hence, higher RMS current stress and losses on the high-side switches are caused (9). For increasing $M_{0}$ values, which are only present for low modulation depths $M_{1}$, the unequal current sharing becomes more pronounced. In this case, however, in many motor drive applications (e.g. compressors) the speed, the EMF and the current amplitude $\hat{I}_{\mathrm{o}}$ of the motor are small and accordingly the unequal distribution of the current stress on both high and low devices is no more critical. On the contrary, the GTHM (i.e. $M_{1}=0, M_{3} \neq 0$ ) modulation equally distributes the current stress among the semiconduc- 
TABLE I

Hardware Demonstrator Specifications

\begin{tabular}{ll}
\hline \hline Parameter & Value \\
\hline Motor & \\
Speed $n$ & $300 \mathrm{krpm}$ \\
Resistance $R_{\mathrm{m}}$ & $0.041 \Omega$ \\
Inductance $L_{\mathrm{m}}$ & $4.72 \mu \mathrm{H}$ \\
Power $P$ & $300 \mathrm{~W}$ \\
Losses $P_{\text {Loss, } \mathrm{m}}$ & $5 \mathrm{~W}$ \\
\hline Inverter & \\
DC-link voltage $V_{\mathrm{DC}}$ & $48 \mathrm{~V}$ \\
Output phase RMS voltage $\tilde{V}_{\mathrm{o}}$ & $19 \mathrm{~V}$ \\
Fundamental frequency $f_{\mathrm{o}}$ & $5 \mathrm{kHz}$ \\
Switching frequency $f_{\mathrm{s}}$ & $140 \mathrm{kHz}$ \\
Filter inductance $L$ & $17 \mu \mathrm{H}$ \\
Filter capacitance $C$ & $1.33 \mu \mathrm{F}$ \\
Power density $\rho$ & $1.91 \mathrm{~kW} / \mathrm{dm}^{3}$ \\
Efficiency $\eta$ & $97.29 \%(\approx 9 \mathrm{~W}$ losses $)$ \\
\hline
\end{tabular}

tors, since the expressions of (9) do not depend on $M_{3}$. The switching performance remains roughly unaffected from the employed modulation scheme, since the same total number of switching transitions occurs independent of the modulation strategy.

The input DC-link capacitor conducts the high frequency switched current $I_{\mathrm{C}}$,in, which in return causes losses in the equivalent series resistance (ESR), and could reduce the life time especially for aluminium electrolytic capacitors. A simple expression relating the capacitor RMS current with the modulation depth $M_{1}$ and the AC-side power factor $\cos (\Phi)$ is given in [17], which is independent of the applied inverter control scheme,

$I_{\mathrm{C}, \mathrm{in}, \mathrm{RMS}}=\frac{\hat{I}_{\mathrm{o}}}{\sqrt{2}} \sqrt{2 M_{1}\left[\frac{\sqrt{3}}{4 \pi}+\cos ^{2}(\phi)\left(\frac{\sqrt{3}}{\pi}-\frac{9}{16} M_{1}\right)\right]}$.

This analytic approximation assumes sinusoidal inverter output currents and thus neglects the marginal increase of the input capacitor current stress caused by the output current ripple (up to $8 \%$ - [17]). Large circulating CM currents, flowing through the DC-link referenced output filter capacitors $C / 2$ in the case of GTHM ((5) - $\left.\hat{I}_{\mathrm{C}, 3}=M_{3} V_{\mathrm{DC}} \pi 3 f_{\mathrm{o}} C\right)$, could further increase the input capacitor RMS current. However, the small capacitance values $C / 2$ typically selected for the implementation of the output filter in order to minimize the fundamental reactive phase current $\hat{I}_{\mathrm{C}, 1}=M_{1} V_{\mathrm{DC}} \pi$ $f_{\mathrm{o}} C \leq 0.1 \hat{I}_{\mathrm{o}}$, inherently limits the CM currents $\hat{I}_{\mathrm{C}, 3}$. Therefore, the effect of the small circulating $\mathrm{CM}$ currents can also be disregarded and the analytic expression (10) can be used for the DC-link capacitor design and loss estimation when any of the discussed modulation schemes SM, DCCMM, GTHM or OCMM is employed.

\section{EXPERIMENTAL VALIDATION}

In order to validate the claimed performance benefits derived from the proposed CM injection techniques, a hardware demonstrator was assembled and tested. Thereby, an industry solu-

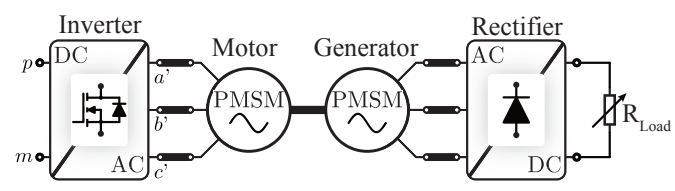

(a)

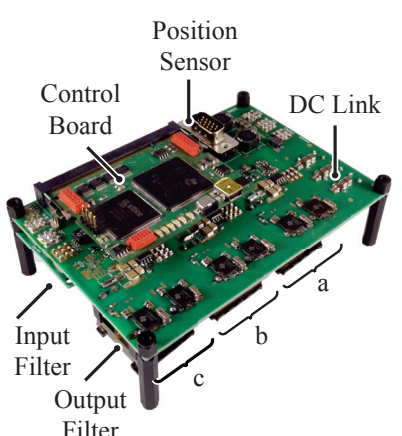

(b)

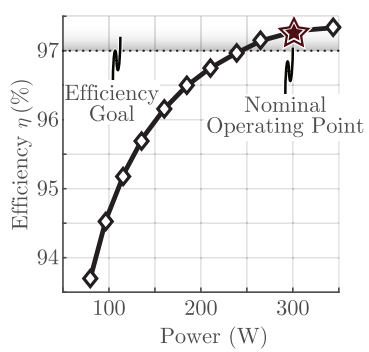

(d)

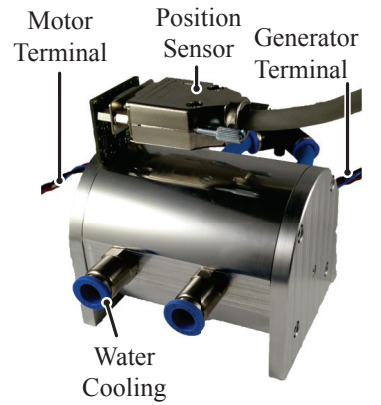

(c)

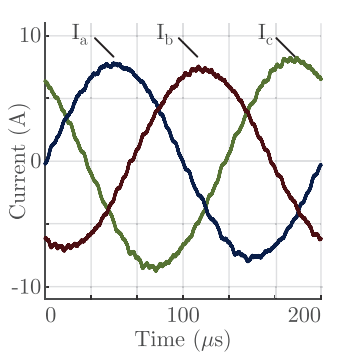

(e)
Fig. 6. An overview of the high-speed motor drive test setup employed for the experimental verification is depicted in (a). In (b), the two-level inverter prototype with a DC-link referenced output filter is shown, while in (c), the constructed ultra-high speed permanent-magnet motor is presented. In (d) the inverter efficiency curve is plotted over the whole power range when sinusoidal modulation (SM) is utilized. In (e), the measured smooth sinusoidal motor currents under nominal speed (i.e. $300 \mathrm{krpm}$ ) and power operation are shown. The slight imbalance of the phase currents originates from asymmetric geometric construction of the phase windings.

tion example of a high-speed motor drive, which features an output power of $300 \mathrm{~W}$ and a rotational motor speed of 300 $\mathrm{krpm}$, is used. The detailed specifications are recapitulated in TABLE I, while the test setup is visualized in Fig. 6(a). The hardware prototype includes a two-level inverter employing the latest generation of $\mathrm{GaN}$ devices and a DC-link referenced output filter as depicted in Fig. 6(b). A two-pole permanentmagnet (PM) slotless design, able to withstand the mechanical stress related to the high rotational speeds and exhibiting very low leakage inductance [18], [19], is selected for the high speed machine implementation. The custom 300 krpm motor prototype (cf. Fig. 6(c)) is built in a back-toback (B2B) configuration. In particular, the inverter driven motor is connected to the same shaft as an identical generator, which subsequently feeds a diode rectifier with an adjustable DC load (cf. Fig. 6(a)). Thereby, the torque and the speed of the motor can be adjusted independently, offering maximum testing flexibility. A cascaded speed and current controller in dq-axis reference frame is implemented using a digital signal processor (DSP) in order to drive the machine. The motor angle $\epsilon$ is provided by a hall sensor board 


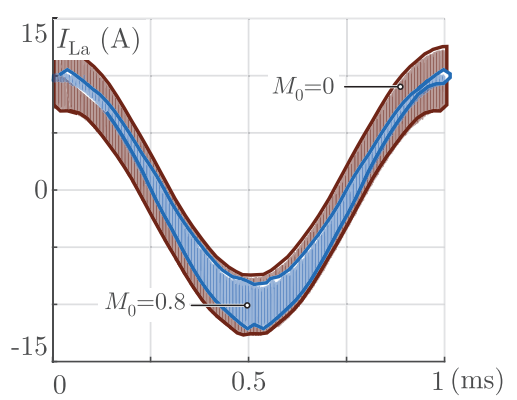

(a.i)

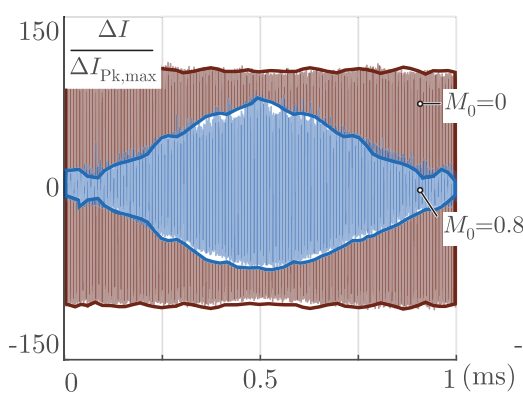

(a.ii)

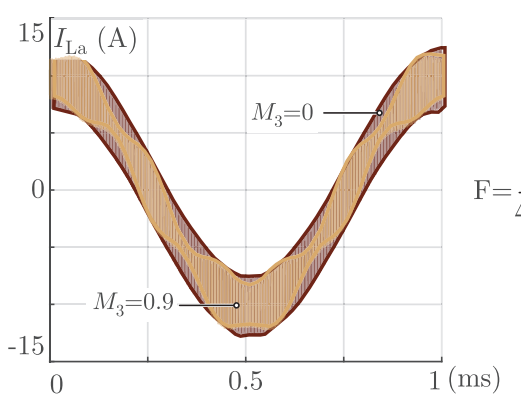

(b.i)

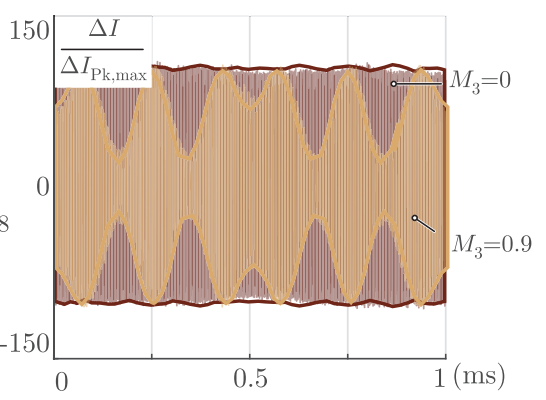

(b.ii)

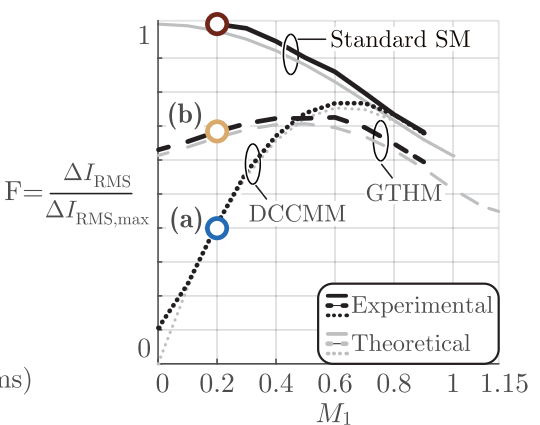

(c)

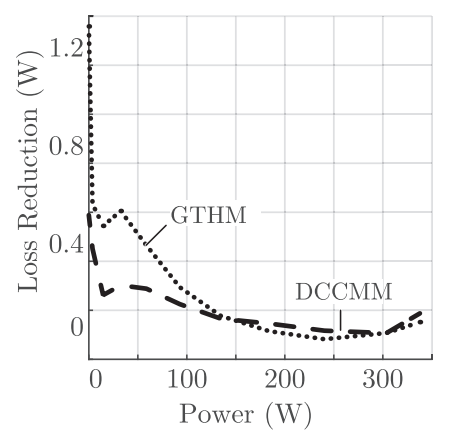

(d)

Fig. 7. In (a.i), the measured inductor currents with $\left(M_{0}=0.8\right)$ and without $\left(M_{0}=0\right)$ DCCMM are depicted. The fundamental frequency is $f_{\mathrm{s}}=1 \mathrm{kHz}$ and the modulation depth is $M_{1}=0.2$. Afterwards, the inductor current ripple is derived by means of data post-processing in (a.ii). The same quantities are plotted in (b.i) and (b.ii) for the same modulation depth $M_{1}=0.2$ when GTHM is employed. In (c), the measured inductor current ripple $\Delta I_{\text {RMS }}$ is compared with its theoretically calculated counterpart (cf. Fig. 5(c)). In (d), the reduction of the losses attained by the two proposed modulation techniques (i.e. DCCMM and GTHM), compared to the standard sinusoidal modulation (SM), is plotted.

that is directly mounted on the machine chassis. Standard sinusoidal modulation (SM) is initially employed in order to commission the inverter-motor combination. The measured inverter efficiency curve and the nominal motor currents at a rotational speed of $300 \mathrm{krpm}$ are shown in Fig. 6(d) and (e) respectively.

In a next step, the two proposed modulation schemes are implemented. The optimal values of the CM injection parameters $M_{0, \text { opt }}$ and $M_{3, \text { opt }}$ corresponding to different modulation depths $M_{1}$ (cf. Fig. 3(a) and Fig. 4(a)) are stored in a lookup table (LUT) within the microcontroller memory and are accessed seamlessly during system operation. Depending on the applied modulation depth $M_{1}$, the optimal injection parameter is directly selected from the LUT or a linear interpolation between the two nearest values is performed. For a motor speed of $60 \mathrm{krpm}$ (i.e. fundamental frequency $f_{\mathrm{s}}=$ $1 \mathrm{kHz}$ ) and the rated motor torque (i.e. rated motor current $10 \mathrm{~A}, M_{1}=0.2$ ), the two proposed methods, DCCMM and GTHM, were tested and the measurement results are plotted in Fig. 7(a),(b). The beneficial shaping of the inductor current ripple envelope is evident for both modulation strategies. Based on the experimental measurements, the RMS inductor current ripple $\Delta I_{\text {RMS }}$ is extracted for different operating points and is compared against the theoretically calculated values. An excellent matching between the theoretical and experimental results is observed in Fig. 7(c). Finally, the inverter losses are measured for multiple power levels $P \in$
[0 W, $350 \mathrm{~W}]$. The achieved loss reduction by employing DCCMM or GTHM, compared to the standard sinusoidal modulation (SM), is shown in Fig. 7(d). The loss reduction is originating exclusively from the minimization of the inductor current ripple. Especially for low modulation depths, where a wider range of $\mathrm{CM}$ injection parameters $M_{0}$ and $M_{3}$ is available (cf. Fig. 3(a) and Fig. 4(a)), the loss benefit is more significant. At the best case, a reduction of the converter losses by $\simeq 1 \mathrm{~W}$ with respect to the nominal converter losses of $9 \mathrm{~W}$ is exhibited.

\section{Conclusions}

Two new modulation techniques, towards highly efficient low-voltage inverters are proposed and analyzed in this paper. The need for new modulation schemes, beyond the stateof-theart solutions, is established for the case of a two-level inverter followed by a DC-link referenced output filter. The degree of freedom, when selecting a modulation technique, is the injected CM voltage pattern $d_{\mathrm{CM}}(t)$. The unique inductor current ripple formation of the DC-link referenced filter motivates the reconsideration of the optimal CM injection waveforms. The proposed constant $\mathrm{CM}$ shifting of the duty cycles (DCCMM) is described first, and afterwards the generalized third harmonic injection modulation (GTHM) is introduced. The main idea behind both schemes lies in the shifting of the inverter switched voltage spectral content 
from the switching frequency towards a low CM modulation frequency (i.e. $f_{\mathrm{CM}}=0$ or $3 f_{\mathrm{o}}$ ). Both methods are evaluated by means of optimization in terms of inductor current ripple. It is concluded that for low modulation depths the DCCMM is more effective, while for high modulation indexes the GTHM yields superior performance. Finally, a benchmark high-speed motor drive is assembled, including a $300 \mathrm{~W}$ converter and $300 \mathrm{krpm}$ motor. The hardware demonstrator is successfully used as framework in order to validate the functionality and effectiveness of the proposed modulations schemes. A loss reduction up to $11 \%$ is attained compared to standard sinusoidal modulation.

\section{ACKNOWLEDGMENT}

The authors gratefully acknowledge the financial support by the Swiss Federal Commission for Technology and Innovation (CTI) and the technical contribution of Celeroton AG.

\section{REFERENCES}

[1] C. Zwyssig, J. W. Kolar, and S. D. Round, "Megaspeed drive systems: pushing beyond 1 million r/min," IEEE/ASME Transactions on Mechatronics, vol. 14, no. 5, pp. 564-574, Oct. 2009.

[2] M. A. Rahman, A. Chiba, and T. Fukao, "Super high speed electrical machines - summary," in Proceedings of IEEE Power Engineering Society General Meeting, Jun. 2004, vol.2, 1272-1275.

[3] J. Muehlethaler, H. Uemura, and J. W. Kolar, "Optimal design of emi filters for single-phase boost pfc circuits," in Proceedings of 38th Annual Conference on IEEE Industrial Electronics Society (IECON), Oct. 2012, pp. 632-638.

[4] D. A. Rendusara and P. N. Enjeti, "An improved inverter output filter configuration reduces common and differential modes $\mathrm{dv} / \mathrm{dt}$ at the motor terminals in pwm drive systems," IEEE Transactions on Power Electronics, vol. 13, no. 6, pp. 1135-1143, Nov. 1998.

[5] C. Marxgut, J. Biela, and J. W. Kolar, "Interleaved triangular current mode (tcm) resonant transition, single-phase pfc rectifier with high efficiency and high power density," in Proceedings of International Power Electronics Conference (ECCE ASIA), Jun. 2010, pp. 17251732.

[6] C. Marxgut, F. Krismer, D. Bortis, and J. W. Kolar, "Ultraflat interleaved triangular current mode (tcm) single-phase pfc rectifier," IEEE Transactions on Power Electronics, vol. 29, no. 2, pp. 873-882, Feb. 2014.

[7] M. Kaufmann, A. Tuysuz, and J. W. Kolar, "New optimum modulation of three-phase zvs triangular current mode gan inverter ensuring limited switching frequency variation," in Proceedings of 8th IET International Conference on Power Electronics, Machines and Drives (PEMD), Apr. 2016, pp. 1-6.

[8] J. W. Kolar, H. Ertl, and F. C. Zach, "Influence of the modulation method on the conduction and switching losses of a pwm converter system," IEEE Transactions on Industry Applications, vol. 27, no. 6, pp. 1063-1075, Nov. 1991.

[9] A. M. Hava, R. J. Kerkman, and T. A. Lipo, "Simple analytical and graphical methods for carrier-based pwm-vsi drives," IEEE Transactions on Power Electronics, vol. 14, no. 1, pp. 49-61, Jan. 1999.

[10] O. Onederra, I. Kortabarria, I. M. de Alegra, J. Andreu, and J. I. Grate, "Three-phase vsi optimal switching loss reduction using variable switching frequency," IEEE Transactions on Power Electronics, vol. 32, no. 8, pp. 6570-6576, Aug. 2017.

[11] D. Neumayr, D. Bortis, E. Hatipoglu, J. W. Kolar, and G. Deboy, "Novel efficiency-optimal frequency modulation for high power density dc/ac converter systems," in Proceedings of 3rd IEEE International Future Energy Electronics Conference (IFEEC - ECCE Asia), Jun. 2017, pp. 834-839.

[12] K. Zhou and D. Wang, "Relationship between space-vector modula- tion and three-phase carrier-based pwm: A comprehensive analysis," IEEE Transactions on Industrial Electronics, vol. 49, no. 1, pp. 186196, Feb. 2002.

[13] A. M. Hava, S. Sul, R. J. Kerkman, and T. A. Lipo, "Dynamic overmodulation characteristics of triangle intersection pwm methods," IEEE Transactions on Industry Applications, vol. 35, no. 4, pp. 896907, Jul. 1999.

[14] F. Liu, K. Xin, and Y. Liu, "An adaptive discontinuous pulse width modulation (dpwm) method for three phase inverter," in Proceedings of IEEE Applied Power Electronics Conference and Exposition (APEC), Mar. 2017, pp. 1467-1472.

[15] C. Charumit and V. Kinnares, "Discontinuous svpwm techniques of threeleg vsi-fed balanced two-phase loads for reduced switching losses and current ripple," IEEE Transactions on Power Electronics, vol. 30, no. 4, pp. 2191-2204, Apr. 2015.

[16] J. W. Kolar, H. Ertl, and F. C. Zach, "Influence of the modulation method on the conduction and switching losses of a pwm converter system," IEEE Transactions on Industry Applications, vol. 27, no. 6, pp. 1063-1075, Nov. 1991.

[17] J. W. Kolar and S. D. Round, "Analytical calculation of the rms current stress on the dc-link capacitor of voltage-pwm converter systems," vol. 153, no. 4, pp. 535-543, Jul. 2006.

[18] A. Tuysuz, A. Schaubhut, C. Zwyssig, and J. W. Kolar, "Model-based loss minimization in high-speed motors," in Proceedings of International Electric Machines Drives Conference (IEMDC), May. 2013, pp. 332-339.

[19] A. Looser, T. Baumgartner, C. Zwyssig, and J. W. Kolar, "Analysis and measurement of $3 \mathrm{~d}$ torque and forces for permanent magnet motors with slotless windings," in Proceedings of IEEE Energy Conversion Congress and Exposition (ECCE USA), Sep. 2010, pp. 37923797.

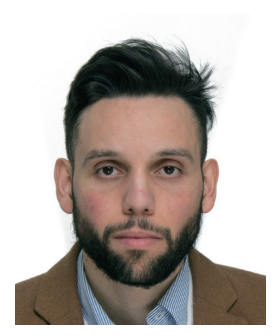

Michael Antivachis received the B.Sc. degree in electrical ngineering from the National Technical University of Athens (NTUA) in 2014 and the M.Sc. degree in energy science and technology from the Swiss Federal Institute of Technology (ETH) in 2016. Since June 2016 he is with the Power Electronic Systems Laboratory (PES) of ETH Zurich as a Ph.D. candidate. His research interests include high-speed motor drive systems for commercial applications, efficient inverter topologies in a small form factor employing wide-bandgap power devices and low EMI emission profile converters.

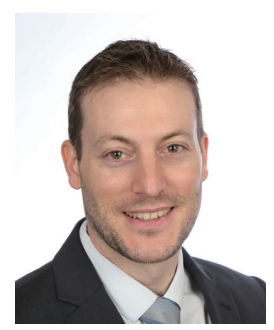

Dominik Bortis received the M.Sc. degree in electrical engineering and the Ph.D. degree from the Swiss Federal Institute of Technology (ETH)Zurich, Switzerland, in 2005 and 2008, respectively. In May 2005, he joined the Power Electronic Systems Laboratory (PES), ETH Zurich, as a Ph.D. student. From 2008 to 2011, he has been a Postdoctoral Fellow and from 2011 to 2016 a Research Associate with PES, co-supervising Ph.D. students and leading industry research projects. Since January 2016 Dr. Bortis is heading the newly established research group Advanced Mechatronic Systems at PES. 


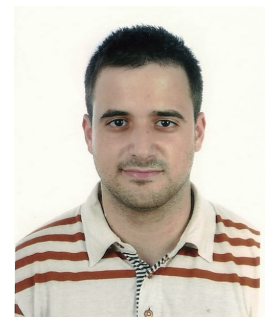

Ander Avila received the B.Sc. degree in industrial electronics and the M.Sc. degree in energy and power electronics from the University of Mondragon, Mondragon, Spain, in 2013 and 2015, respectively. Since 2015, he has been a Researcher with the IK4-Ikerlan Technology Research Centre, Mondragon, where he has been pursuing his $\mathrm{Ph} . \mathrm{D}$. in collaboration with the Power Supply System Group, University of Oviedo, Gijon, Spain. His research interests include wide band-gap semiconductors and the design, control and optimization of hard and soft-switching power converters for railway, storage systems and inductive power transfer applications.

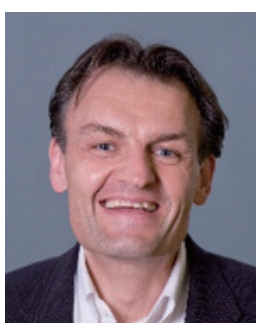

Johann W. Kolar is a Fellow of the IEEE and received his $\mathrm{Ph} . \mathrm{D}$. degree (summa cum laude) from the Vienna University of Technology, Austria. He is currently a Full Professor and the Head of the Power Electronic Systems Laboratory at the Swiss Federal Institute of Technology (ETH) Zurich. He has published over 750 scientific papers in international journals and conference proceedings and has filed more than 140 patents. He has presented over 20 educational seminars at 1eading international conferences, has served as IEEE PELS Distinguished Lecturer from 2012 through 2016, and has received 25 IEEE Transactions and Conference Prize Paper Awards, the 2014 IEEE Power Electronics Society R. David Middlebrook Achievement Award, the 2016 IEEE William E. Newell Power Electronics Award, the 2016 IEEE PEMC Council Award and the ETH Zurich Golden Owl Award for excellence in teaching. The focus of his current research is on ultra-compact and ultra-efficient $\mathrm{SiC}$ and $\mathrm{GaN}$ converter systems, wireless power transfer, Solid-State Transformers, Power Supplies on Chip, as well as ultra-high speed and ultra-light weight drives, bearingless motors, and energy harvesting. 